\title{
Embryo integrity regulates maternal proteostasis and stress resilience
}

\author{
Ambre J. Sala, Laura C. Bott, Renee M. Brielmann, and Richard I. Morimoto \\ Department of Molecular Biosciences, Rice Institute for Biomedical Research, Northwestern University, Evanston, \\ Illinois 60208, USA
}

\begin{abstract}
The proteostasis network is regulated by transcellular communication to promote health and fitness in metazoans. In Caenorhabditis elegans, signals from the germline initiate the decline of proteostasis and repression of cell stress responses at reproductive maturity, indicating that commitment to reproduction is detrimental to somatic health. Here we show that proteostasis and stress resilience are also regulated by embryo-to-mother communication in reproductive adults. To identify genes that act directly in the reproductive system to regulate somatic proteostasis, we performed a tissue targeted genetic screen for germline modifiers of polyglutamine aggregation in muscle cells. We found that inhibiting the formation of the extracellular vitelline layer of the fertilized embryo inside the uterus suppresses aggregation, improves stress resilience in an HSF-1-dependent manner, and restores the heat-shock response in the somatic tissues of the parent. This pathway relies on DAF-16/FOXO activation in vulval tissues to maintain stress resilience in the mother, suggesting that the integrity of the embryo is monitored by the vulva to detect damage and initiate an organismal protective response. Our findings reveal a previously undescribed transcellular pathway that links the integrity of the developing progeny to proteostasis regulation in the parent.
\end{abstract}

[Keywords: embryo; heat shock response; protein homeostasis; stress resistance; transcellular regulation]

Supplemental material is available for this article.

Received November 25, 2019; revised version accepted March 10, 2020.

Proteostasis relies on an extended network of protective machineries composed of molecular chaperones and protein degradation pathways that together prevent the accumulation of misfolded and aggregated proteins (Sala et al. 2017; Hipp et al. 2019). When proteostasis capacity becomes limited, the presence of misfolded proteins can activate cell stress responses, such as the heat-shock response (HSR), which induce the expression of proteostasis network (PN) components (Korennykh and Walter 2012; Li et al. 2017; Shpilka and Haynes 2018). In multicellular organisms, cell stress responses are controlled by transcellular communication to allow the coordinated regulation of $\mathrm{PN}$ components and promote organismal proteostasis and fitness. During heat stress, the HSR is controlled by the thermosensory neuronal circuitry in C. elegans (Prahlad et al. 2008). Localized stress or imbalances in specific tissues, such as changes in the expression level of a molecular chaperone, constitutive activation of a cell stress response, or perturbation of mitochondrial function, can trigger compensatory cell-nonautonomous responses that lead to the activation of PN components in distinct tissues (Owusu-Ansah et al. 2013; Taylor and Dillin 2013; van Oosten-Hawle et al. 2013; Williams et al. 2014).

Corresponding author: r-morimoto@northwestern.edu Article published online ahead of print. Article and publication date are online at http://www.genesdev.org/cgi/doi/10.1101/gad.335422.119. Freely available online through the Genes \& Development Open Access option.
The reproductive system has an important role in the cell-nonautonomous regulation of organismal proteostasis and health. The functionality of the PN declines in early aging in $C$. elegans, resulting in protein misfolding and aggregation (Ben-Zvi et al. 2009; Walther et al. 2015). This event is preceded by the repression of the HSR and other cell stress responses at the onset of reproduction, which is initiated by signals from the germline stem cells (GSC) (Shemesh et al. 2013; Labbadia and Morimoto 2015). Removal of the GSC prevents this repression and leads to improved stress resilience, reduced protein aggregation, and increased life span (Hsin and Kenyon 1999; Flatt et al. 2008; Shemesh et al. 2013). Therefore, cell-nonautonomous regulation by the reproductive system initiates a program that prioritizes the generation of healthy progeny over maintenance of somatic proteostasis in the reproductive adult.

To obtain further insight into the regulation of proteostasis by the reproductive system, we performed a genetic screen for germline-specific modifiers of somatic proteostasis in C. elegans. We found that inhibiting key components of the vitelline layer-the extracellular coat that surrounds the developing embryo-activates a transcellular pathway that prevents protein aggregation and restores

(C) 2020 Sala et al. This article, published in Genes \& Development, is available under a Creative Commons License (Attribution-NonCommercial 4.0 International), as described at http://creativecommons.org/licenses/by-nc/4.0/. 
the HSR in the mother. This pathway relies on DAF-16 in the vulva and HSF-1 in somatic tissues to promote stress resilience in reproductive adults. Our findings reveal a transcellular pathway that regulates maternal proteostasis when embryo integrity is compromised.

\section{Results}

Inhibition of the germline-specific gene cbd-1 enhances proteostasis in somatic tissues

The reproductive system is an important regulator of organismal proteostasis. To identify cell-nonautonomous pathways that act in germline tissues to distantly control somatic proteostasis, we developed a screening strategy based on existing strains for tissue targeted RNA interference (RNAi) and monitored proteostasis in muscle cells of C. elegans (Espelt et al. 2005; Marré et al. 2016). Using an aggregation-prone polyglutamine (polyQ) peptide fused to yellow fluorescent protein (Q35::YFP) expressed in bodywall muscle as a readout of proteostasis (Supplemental Fig. S1A-D), we screened a library of 93 suppressors of Q35::YFP aggregation previously identified in a systemic RNAi screen (Silva et al. 2011) for modifiers that act in the germline to improve proteostasis in muscle (see Materials and Methods for details). This strategy identified six germline-specific candidates that reduced the number of Q35::YFP foci in body-wall muscle (Supplemental Fig. $\mathrm{S} 1 \mathrm{E}, \mathrm{F}$ ). Since loss of GSC enhances proteostasis (Ben-Zvi et al. 2009; Shemesh et al. 2013; Labbadia and Morimoto 2015), we assessed the effect of these candidate genes on the number of cells in the mitotic region of the germline to exclude those genes whose knockdown alters GSC homeostasis. Five of the germline-specific candidates correspond to genes involved in essential processes and knockdown caused defects in the mitotic region of the germline (Supplemental Fig. S1G,H), whereas the sixth gene, $c b d-1$ (chitin-binding domain protein 1), showed no detectable effect on the number of cells in the mitotic zone of the germline upon RNAi (Supplemental Fig. S1H, I). We concluded that $c b d-1$ inhibition enhances muscle proteostasis via a mechanism that is distinct from GSC loss.

In metazoans, the developing embryo is surrounded by an extracellular coat that forms around the egg following fertilization, providing physical protection to the embryo and functioning as the interface with the environment (Olson et al. 2012). In C. elegans, this coat is known as the eggshell and $c b d-1$ encodes a key component of the outermost layer of the eggshell, the vitelline layer (Johnston et al. 2010). As cbd-1 is a germline-specific gene (Johnston et al. 2010), we used systemic RNAi for the rest of our study. Knockdown of $c b d-1(90 \%$ reduction in $c b d-1$ mRNA levels; Supplemental Fig. S1J) suppressed the age-dependent accumulation of Q35::YFP aggregates in muscle cells by $20 \%-40 \%$ compared with the empty vector control (Fig. 1A; Supplemental Figs. S1F, S2A). To examine the effect of $c b d-1$ RNAi on the folding and function of an endogenous protein, we used a strain harboring a temperature-sensitive allele of the myosin heavy chain
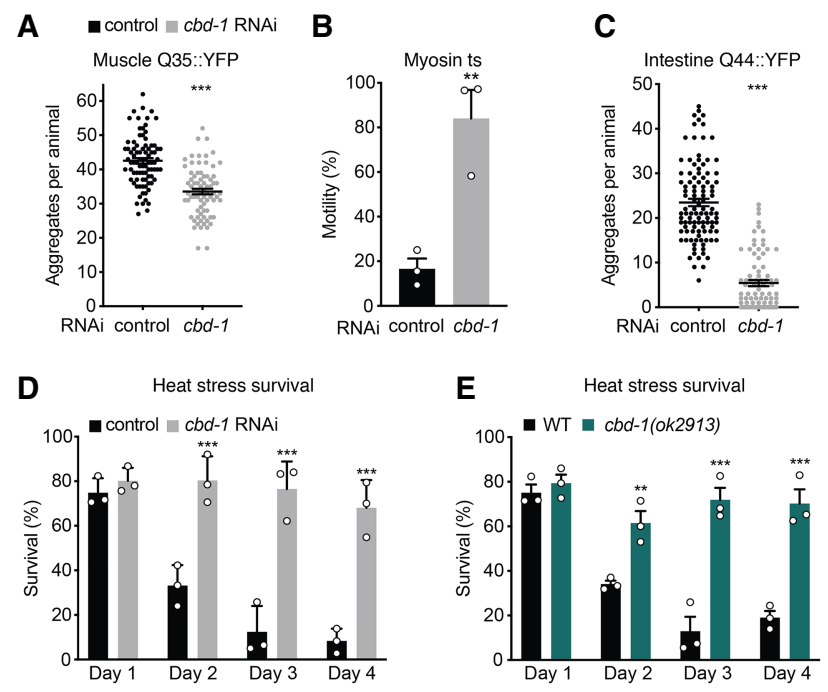

Figure 1. Inhibition of $c b d-1$ enhances somatic proteostasis and stress resilience in reproductive adults. (A) Quantification of muscle Q35::YFP aggregates in day 4 adults treated with either control (empty vector) or $c b d-1$ RNAi. Control, $n=86$; $c b d-1$ RNAi, $n=76$. (B) Motility of unc-54(e1301) temperature-sensitive (ts) mutants at $25^{\circ} \mathrm{C}(n=3,35$ animals per trial). (C) Quantification of intestinal Q44::YFP aggregates in day 5 adults. Control, $n=105$; $c b d-1$ RNAi, $n=84$. (D) Heat stress survival of wildtype animals exposed to a $35^{\circ} \mathrm{C}$ heat shock on the indicated days of adulthood and allowed to recover for $48 \mathrm{~h}$ at $20^{\circ} \mathrm{C}(n=$ 3). (E) Heat stress survival of wild-type (WT) or cbd-1(ok2913) animals $(n=3)$. Error bars represent SEM. Statistical significance based on unpaired $t$-test $(A-C)$ or two-way ANOVA with Sidak correction and pairwise comparisons relative to control RNAi $\left.\left.(D-E) .{ }^{* *}\right) P<0.01 ;{ }^{* * *}\right) P<0.001$. For $A$ and $C, n$ is total number of animals analyzed in three independent experiments. For $B$, $D$, and $E, n$ denotes biological replicates of population analysis.

gene unc-54 that causes paralysis when the mutant UNC-54 protein misfolds (Gidalevitz et al. 2006). Motility of unc-54(e1301) animals was improved fourfold in $c b d-1$ RNAi-treated animals compared with control (Fig. 1B). To determine whether the beneficial effects of $c b d-1$ knockdown extend to other somatic tissues, we used an intestinal polyQ model and found that animals treated with $c b d$ 1 RNAi showed a 75\% reduction in Q44::YFP foci compared with control (Fig. 1C; Supplemental Fig. S2B). These results indicate that inhibition of the vitelline layer component CBD-1 suppresses protein misfolding and aggregation in multiple somatic tissues of the parent.

\section{Inhibition of cbd-1 restores stress resilience and the heat-shock response in reproductive adults}

Resistance to heat stress in C. elegans decreases dramatically during the first day of adulthood when animals begin to produce progeny (Labbadia and Morimoto 2015). We thus asked whether $c b d-1$ inhibition could also modulate stress resilience in reproductive adults, and exposed animals treated with $c b d-1$ or control RNAi to acute heat shock on different days of adulthood. As expected, control animals exhibited a $50 \%$ decline in heat stress survival 
after day 1 that extended to an $85 \%$ decline in viability on day 4 of adulthood (Fig. 1D). In contrast, cbd-1 RNAitreated animals maintained elevated levels of heat stress survival through day 4 of adulthood compared with prereproductive day 1 adults (Fig. 1D). We confirmed these observations in animals harboring the cbd-1(ok2913) hypomorph allele (Supplemental Fig. S2C; González et al. 2018), which exhibited elevated heat stress survival in adulthood to levels similar to $c b d-1$ RNAi (Fig. 1E). Notably, $c b d-1$ inhibition had no effect on the stress resilience of prereproductive day 1 animals (Supplemental Fig. S2D), or in mutants lacking gonads (Supplemental Fig. S2E), in agreement with the function of the $c b d-1$ gene product in eggshell formation. Collectively, these results indicate that inhibition of $c b d-1$ restores stress resilience in reproductive adults.

Repression of the HSR at the transition to reproductive maturity underlies reduced stress resilience (Labbadia and Morimoto 2015). To test whether maintained stress resilience in adulthood upon $c b d-1$ RNAi is accompanied by elevated HSR, we monitored the expression level of three inducible heat-shock genes corresponding to $h s p-70$ (C12C8.1), hsp-70(F44E5.4), and hsp-16.11, following heat shock. As expected, heat-inducible expression of all three genes declined by $\sim 60 \%$ between day 1 and day 2 of adulthood in animals grown on control RNAi (Fig. $2 \mathrm{~A}-\mathrm{C})$. In contrast, animals grown on $c b d-1$ RNAi maintained the expression of heat shock genes through day 4 of adulthood. Basal expression of heat-shock genes was

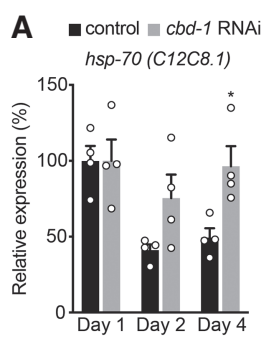

D

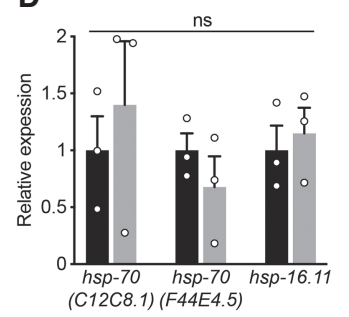

C
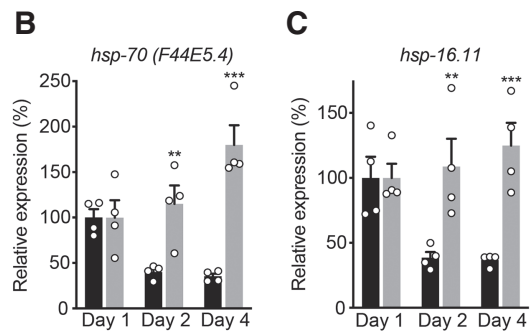

E

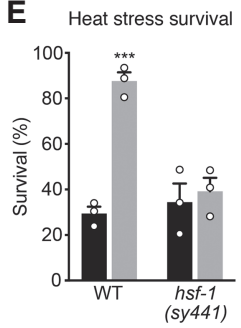

Figure 2. Inhibition of $c b d-1$ restores the heat-shock response in reproductive adults. $(A-C)$ Expression of heat-shock genes relative to $c d c-42$ in wild-type animals exposed for $30 \mathrm{~min}$ to $33^{\circ} \mathrm{C}$ heat shock on the indicated days of adulthood $(n=4)$. (D) Basal expression of heat-shock genes relative to $c d c-42(n=3)$. $(E)$ Heat stress survival of wild-type (WT) or hsf-1(sy441) day 2 adults $(n=3)$. Error bars represent SEM. Statistical significance based on two-way ANOVA with Sidak correction and pairwise comparisons relative to control RNAi $(A-D)$ or multiple $t$-tests with Holm-Sidak correction $\left.(E) .\left(^{*}\right) P<0.05 ;\left({ }^{* *}\right) P<0.01 ;{ }^{* * *}\right) P<0.001$; (ns [nonsignificant]) $P>0.05 . n$ denotes biological replicates of population analysis. unchanged upon cbd-1 RNAi (Fig. 2D; Supplemental Fig. S2F), indicating that the induction of the HSR was selectively affected. We therefore asked whether the beneficial effects of $c b d-1$ inhibition on stress resilience required HSF-1, the central regulator of the HSR that controls organismal stress resistance and cellular proteostasis (Morley and Morimoto 2004), and found that the elevated heat stress resistance upon $c b d-1$ knockdown was completely suppressed in the hsf-1(sy441) mutant background (Fig. 2E). Thus, inhibition of $c b d-1$ acts in an HSF-1-dependent manner to improve stress resilience and restore the HSR in reproductive adults.

\section{Impairment of the vitelline layer of fertilized embryos in the uterus enhances maternal stress resilience and proteostasis}

CBD-1 is secreted in oocytes and incorporated into the vitelline layer of the eggshell (Johnston et al. 2010; González et al. 2018). To visualize the expression pattern of the CBD-1 protein, we introduced an enhanced green fluorescent protein (eGFP) tag upstream of the stop codon of $c b d-1$ using CRISPR-Cas9 to generate CBD-1::eGFP (Supplemental Fig. S3A,B). As expected, CBD-1::eGFP expression was restricted to the germline of adult animals (Fig. 3A), and specifically detected at the periphery of maturing oocytes and fertilized eggs (Fig. 3B,C). cbd-1 RNAi resulted in a loss of structural integrity of fertilized eggs, while the rest of the germline seemed unaffected (Fig. 3D; Johnston et al. 2010). Consistently, we found that $c b d-1$ inhibition by either RNAi or in the cbd-1(ok2913) mutant reduced brood size by $>95 \%$ and $80 \%$ respectively, in agreement with previous findings that loss of eggshell integrity is deleterious to embryo viability (Supplemental Fig. S3B,C; Johnston et al. 2006; González et al. 2018). To determine whether the beneficial effects of $c b d-1$ inhibition require fertilized eggs, we used the fog-2( $q 71)$ mutant in which hermaphrodites produce oocytes but not sperm and therefore lack fertilized eggs unless they are mated to males (Schedl and Kimble 1988). Unmated fog-2(q71) hermaphrodites grown on $c b d-1$ RNAi exhibited levels of heat stress survival similar to control RNAi (Fig. 3E). In contrast, mated fog-2 mutants showed enhanced heat stress survival upon $c b d-1$ knockdown, similar to wildtype animals (Fig. 3E). These results indicate that the presence of oocytes is insufficient for $c b d-1$ inhibition to improve organismal stress resilience and that fertilized eggs are required for this effect. Together, these results suggest that loss of vitelline membrane integrity inside the uterus is sensed by the mother to trigger a systemic protective response.

Given the link between CBD-1 and somatic proteostasis, we next asked whether this observation extends to other components of the eggshell. CBD-1 forms a complex with PERM-2 and PERM-4 in the vitelline layer, which is established first and corresponds to the outermost layer of the eggshell (Fig. 3F; González et al. 2018). Like CBD-1, PERM-2 and PERM-4 are found on the surface of oocytes in the gonad before fertilization and on the surface of the embryo after fertilization, and knockdown results in a loss 
A

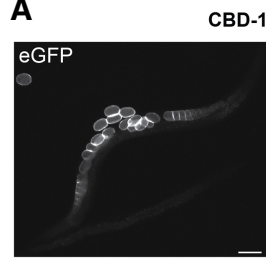

E Heat stress survival 100 control $1=c b d-1$ RNAi

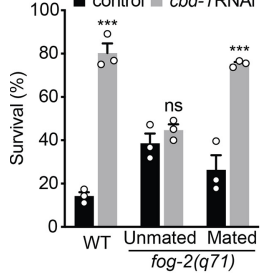

CBD-1::eGFP

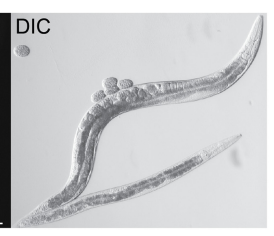

F Fertilized egg

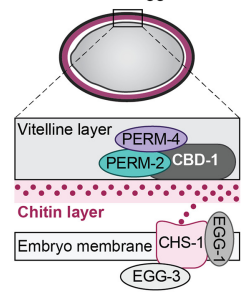

B Mitotic zone

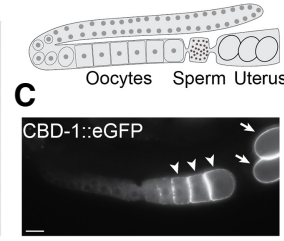

G
D

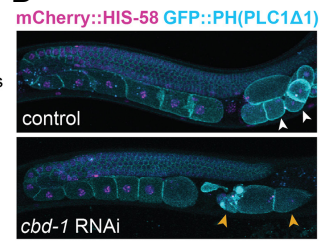

H Intestine Q44::YFP

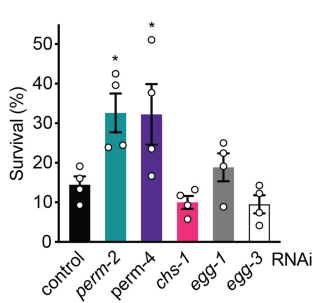

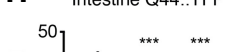

Figure 3. Impairment of the vitelline layer of fertilized embryos in the uterus enhances maternal stress resilience and proteostasis. (A) Fluorescence and DIC images showing a gravid adult and an L4 stage larvae harboring the $c b d-1:: e g f p$ allele. Scale bar, $100 \mu \mathrm{m}$. (B) Schematic structure of the C. elegans gonad. (C) Epifluorescence image showing one gonad arm of a gravid adult expressing CBD1::eGFP. Arrowheads indicate unfertilized oocytes and arrows indicate fertilized eggs. Scale bar, $20 \mu \mathrm{m}$. $(D)$ Confocal images showing one gonad of gravid day 1 adults expressing fluorescent markers for the plasma membrane [GFP::PH(PLC $\Delta 1) ;$ cyan] and chromosomes (mCherry::HIS-58; magenta) in the germline. White arrowheads indicate normal eggs in the uterus and orange arrowheads indicate eggs with abnormal shape. (E) Heat stress survival of day 3 adult hermaphrodites. fog-2(q71) were either separated from males at L4 larval stage (unmated) or not (mated) $(n=3)$. ( $F)$ Schematic of the eggshell following fertilization. $(G)$ Heat stress survival of wild-type day 3 adults grown on indicated RNAi $(n=4)$. $(H)$ Quantification of intestinal Q44::YFP aggregates in day 5 wild-type adults grown on indicated RNAi. Control, $n=105$; perm-2 RNAi, $n=99$; perm-4 RNAi, $n=88$. Error bars represent SEM. Statistical significance based on multiple $t$-tests with Holm-Sidak correction $(E)$ or one-way ANOVA with Dunnett correction relative to control RNAi $(G, H) .\left({ }^{*}\right) P<0.05 ;\left(^{* * *}\right) P<0.001$; (ns [nonsignificant]) $P>0.05$. For $E$ and $G, n$ denotes biological replicates of population analysis, while in $H, n$ is the total number of animals analyzed in three independent experiments.

of structural integrity of fertilized eggs (González et al. 2018). We found that perm-2 and perm-4 RNAi also increased survival following heat stress, whereas RNAi of chs-1, egg-1, or egg-3, which are involved in the formation of the chitin layer of the eggshell that is produced following fertilization (Fig. 3F; Maruyama et al. 2007), had no effect (Fig. 3G), suggesting that modulation of stress resilience upon disruption of the eggshell is specific to components of the vitelline layer. We next examined whether inhibition of perm-2 and perm-4 also restores somatic proteostasis and found that RNAi of either perm-2 or perm-4 suppressed the age-dependent aggregation of intestinal Q44::YFP by $>50 \%$ (Fig. $3 \mathrm{H}$; Supplemental Fig. S3D). The impact of perm-2 and perm-4 RNAi on brood size was less severe than cbd-1 RNAi (Supplemental Fig. S3C), consistent with the lower effect of knockdown of these genes on stress resilience and polyQ aggregation. Thus, damage specifically to the vitelline layer surrounding the embryo initiates a response that promotes maternal stress resilience and prevents aggregation in somatic tissues.

\section{cbd-1 inhibition triggers a DAF-16 response specifically in the vulva}

Similar to the systemic response caused by damage to the vitelline layer observed here, GSC removal also prevents the decline of the HSR and somatic proteostasis (Shemesh et al. 2013; Labbadia and Morimoto 2015), and therefore we asked whether they act via the same pathway. GSC loss activates the expression of a set of genes involved in diverse processes including stress resistance, fat metabolism, and protein degradation (Antebi 2013). We examined the expression of representative genes of these pathways previously shown to be induced by GSC loss corresponding to sod-3, gst-4, lgg-1, fat-6, and fard-1. We found that only sod-3 was constitutively induced (approximately threefold), both by $c b d-1$ RNAi and in the $c b d-1$ (ok2913) mutant (Fig. 4A; Supplemental Fig. S4A), suggesting that the organismal response upon damage to the vitelline layer of the embryo is distinct from the GSC pathway. Furthermore, whereas GSC ablation extends life span (Hsin and Kenyon 1999), the cbd-1 mutation did not extend life span beyond that of wild-type animals (Supplemental Fig. S4B).

The finding of selective induction of sod-3 following $c b d-1$ inhibition led us to investigate the role of DAF-16, which is known to bind directly to the promoter region of sod-3 to regulate its transcription (Oh et al. 2006). Induction of sod-3 upon cbd-1 RNAi was abolished in the lossof-function daf-16(mu86) mutant (Fig. 4B). We found that expression levels of other known DAF-16 targets dod-8, gpd-2, hsp-12.6, and hsp-16.2 (Zhang et al. 2013) were not affected by $c b d-1$ knockdown or $c b d-1$ (ok29313) hypomorph mutation (Fig. 4C; Supplemental Fig. S4C). As these targets are known to be regulated by DAF-16 in the context of reduced insulin/IGF-1 signaling (IIS) in a daf-2 mutant background (Zhang et al. 2013), these results suggest that $c b d-1$ knockdown induces a DAF-16 program that is distinct from IIS. Interestingly, expression of an sod-3p::GFP reporter was also induced upon $c b d-1$ RNAi and in the cbd-1(ok2913) mutant, and its expression appeared to be restricted to the vulva (Fig. 4D-F; Supplemental Fig. S5A), as confirmed by cross-referencing to vulva-specific markers (Inoue et al. 2002). Importantly, this expression pattern differs from the systemic sod-3 induction observed in the daf-2(e1370) mutant in which DAF-16 is activated in most tissues (Supplemental Fig. S5B; Libina et al. 2003). 

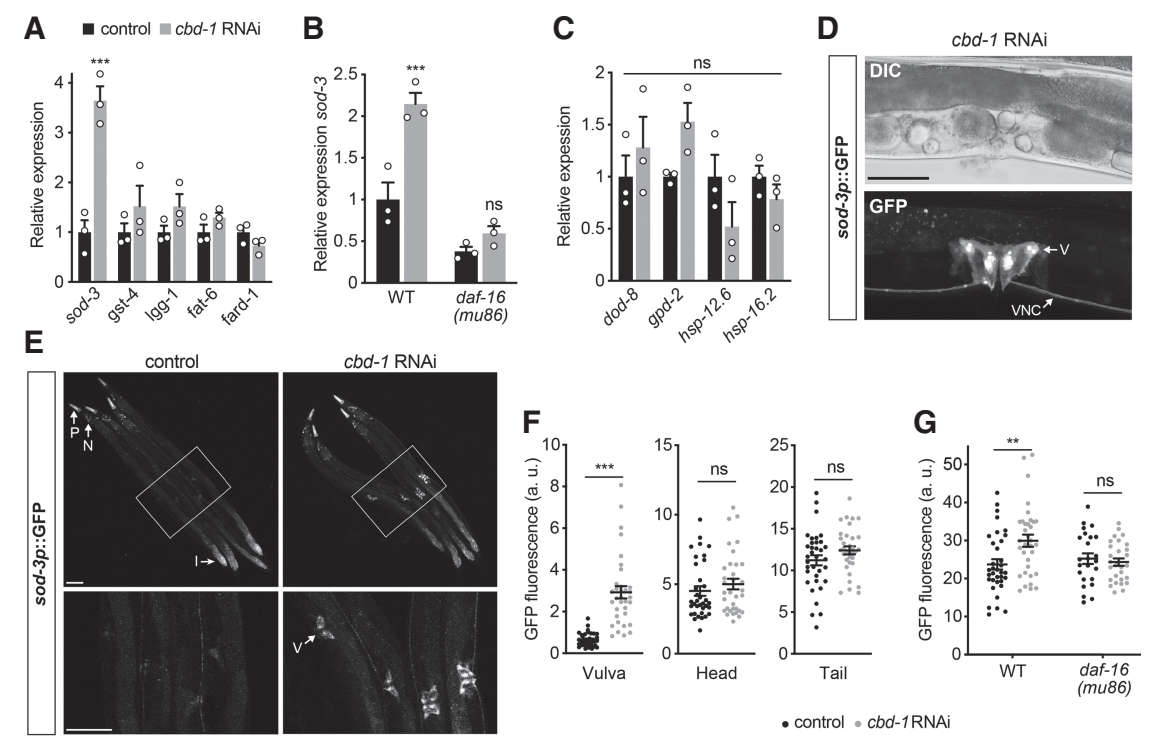

Figure 4. $c b d-1$ inhibition triggers a DAF16 response specifically in the vulva. (A) Expression of indicated genes relative to $c d c$ 42 in wild-type day 2 adults normalized to control RNAi $(n=3)$. (B) Expression of sod3 relative to $c d c-42$ in day 2 adults normalized to wild-type animals grown on control RNAi $(n=3) .(C)$ Expression of indicated genes relative to $c d c-42$ in wild-type day 2 adults normalized to control RNAi $(n=3)$. $(D, E)$ Representative confocal images of day 2 adults expressing the sod-3p::GFP reporter. Arrows indicate the tissues in which the reporter is detected. (V) Vulva; (VNC) ventral nerve cord; $(\mathrm{P})$ pharynx; $(\mathrm{N})$ nerve ring; (I) intestine. Scale bars: $D, 50 \mu \mathrm{m} ; E$, $100 \mu \mathrm{m}$. (F) Quantification of the sod-3p:: GFP reporter fluorescence in the indicated regions of wild-type animals (see the Material and Methods for details). Control, $n=$ 35; $c b d-1$ RNAi, $n=34$ (G) Quantification of $\operatorname{sod}-3 p::$ GFP in whole animals. WT control, $n=35$; WT $c b d-1$ RNAi, $n=34$; daf-16(mu86) control, $n=24$; daf-16(mu86) $c b d-1$ RNAi, $n=28$. Error bars represent SEM. Statistical significance based on two-way ANOVA followed by Sidak correction and pair-wise comparisons with control RNAi $(A, C)$, multiple $t$-tests with Holm-Sidak correction $(B)$, or unpaired $t$-test $(F, G) .\left(^{* *}\right) P<0.01$; $\left(^{* * *}\right) P<0.001$; (ns [nonsignificant]) $P>0.05$. In $A-C, n$ denotes biological replicates of population analysis, and in $F$ and $G, n$ is the total number of animals analyzed in three independent experiments.

The expression of sod-3 was further induced by $c b d-1$ knockdown in daf-2(e1370) mutant, further suggesting that the response is independent from the IIS pathway (Supplemental Fig. S5C,D). Reporter induction upon $c b d-1$ knockdown was not detected in the daf-16(mu86) mutant (Fig. 4G; Supplemental Fig. S5E), indicating that DAF-16 is required for the vulva-specific induction of sod-3 expression in response to $c b d-1$ inhibition.

DAF-16 is required in the vulva for the beneficial effects of vitelline damage on organismal stress resilience

Given the tissue selectivity of sod-3 induction, we hypothesized that the vulva-specific DAF-16 response is essential for embryo-to-mother communication following damage to the vitelline layer. To test this, we asked whether the vulva is required for the beneficial effects of $c b d-1$ inhibition on organismal stress resistance. The development of the vulva has been studied extensively in C. elegans, and many mutants defective in vulva induction have been characterized (Sternberg and Horvitz 1986; Sharma-Kishore et al. 1999; Sherwood and Sternberg 2003; Gupta et al. 2012). Vulvaless mutants produce viable progeny but are unable to lay eggs and die prematurely as a result of internal hatching (Ferguson and Horvitz 1985; Sternberg and Horvitz 1989|. We examined the effect of $c b d-1$ knock-down on the stress resilience of two vulvaless mutants, lin-2(e1309) and lin-7(n308) (Simske et al. 1996), in reproductive day 1 adults that are past the collapse of stress resilience (Labbadia and Morimoto 2015) but do not yet exhibit internal hatching. In both mutants, elevated heat stress survival was completely suppressed upon $c b d-1$ RNAi (Fig. 5A).

We next asked whether DAF-16 is required for the protective effects of $c b d-1$ inhibition and found that the ele- vated heat stress survival upon $c b d-1$ RNAi was abolished in the daf-16(mu86) mutant (Fig. 5B). We further asked whether the maintained HSR in adulthood upon $c b d-1$ inhibition also relies on DAF-16. The expression of both $h s p-70$ (F44E5.4) and $h s p-16.11$ following heat shock was strongly reduced on the empty vector control as compared with day 1 young adults for both the wildtype and daf-16(mu86) mutant, as expected (Fig 5C,D). Upon $c b d-1$ RNAi, the expression of both heat-shock genes was reduced in daf-16(mu86) compared with wildtype animals, although the response was not completely abolished in the daf-16 mutant compared with the empty vector control (Fig. 5C,D). In order to demonstrate that DAF-16 is required in the vulva for the beneficial effects of vitelline damage on organismal stress resilience, we introduced the deletion corresponding to the $c b d-1$ (ok2913) allele into a strain in which RNAi is restricted to the vulva precursor cells (Barkoulas et al. 2013) that differentiate to form vulval tissue in the adult (Gupta et al. 2012). As expected, the resulting strain exhibited elevated stress resistance compared with the parental strain carrying the wildtype $c b d-1$ allele. We found that daf-16 RNAi abolished such elevated heat stress resistance, similar to the $c b d-1$ (ok2913) strain with systemic RNAi (Fig. 5E). Collectively, these results suggest that DAF- 16 activation in the vulva is required to elicit the protective systemic response upon loss of embryo integrity.

\section{Discussion}

The germline initiates the repression of cellular stress responses and the subsequent decline of proteostasis at reproductive maturity in C. elegans (Shemesh et al. 2013; Labbadia and Morimoto 2015), indicating that 


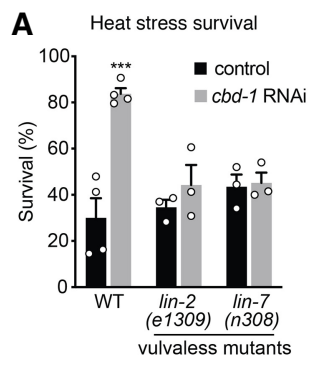

B Heat stress survival
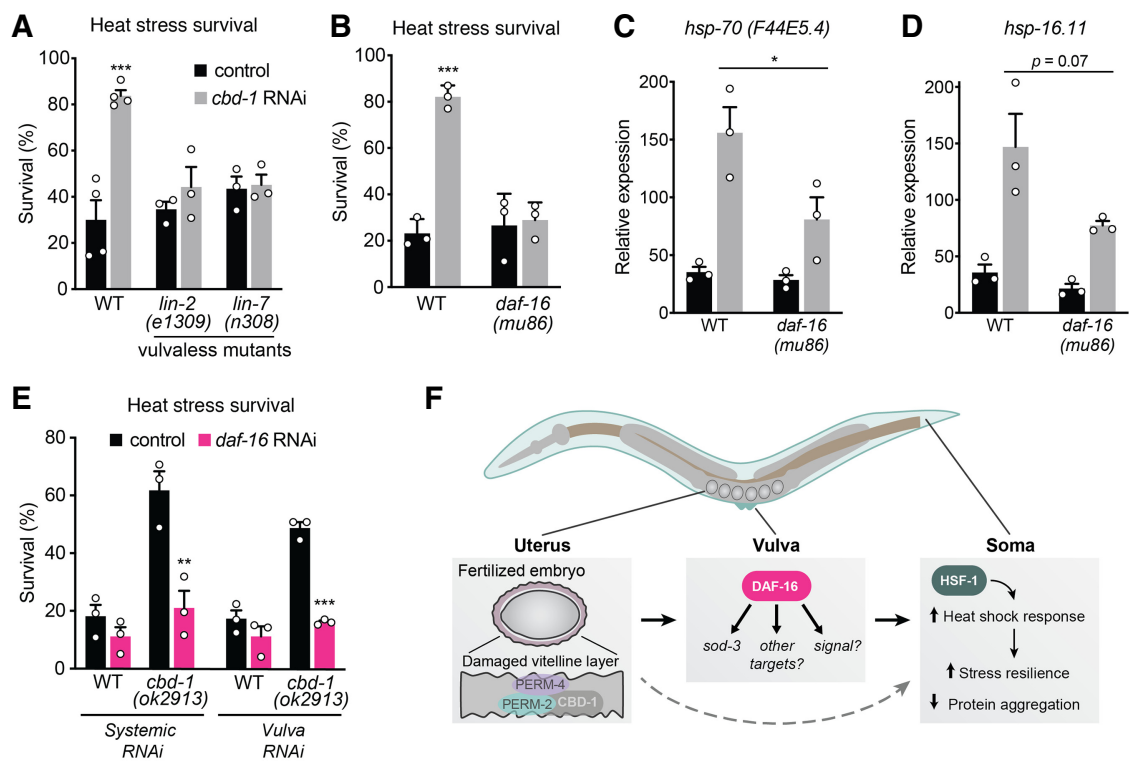

$\mathbf{F}$

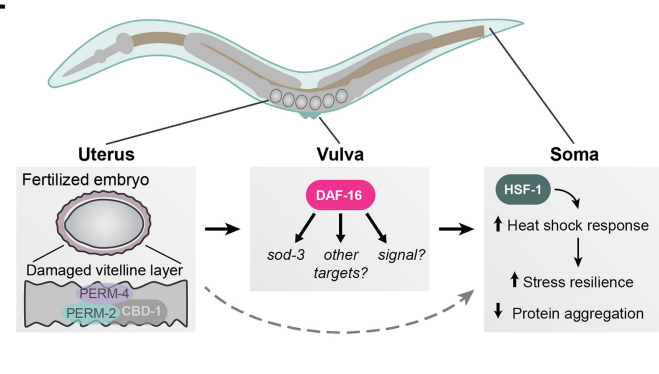

Figure 5. DAF-16 is required in the vulva for the beneficial effects of vitelline damage on organismal stress resistance $(A)$ Heat stress survival of gravid day 1 adults $(n=3)$. (B) Heat stress survival of day 3 adults $(n=$ 3). $(C, D)$ Expression of heat-shock genes relative to $c d c-42$ in wild-type or daf-16(mu86) mutant day 3 adults exposed for $30 \mathrm{~min}$ to $33^{\circ} \mathrm{C}$ heat shock, normalized for each strain to expression at day 1 on control RNAi $(n=$ 3). (E) Heat stress survival of wild-type (systemic RNAi) or vulva precursor cell-specific RNAi strain JU2039 (vulva RNAi) with either the wild-type $c b d-1$ allele (WT) or the $c b d-1$ allele corresponding to the $c b d-1$ (ok2913) deletion. Animals were grown on either control or daf-16 RNAi and heat shocked on day 3 of adulthood $(n=3)$. (F) Model for the embryo-to-mother transcellular pathway. In reproductive adults, perturbation of the vitelline layer of the fertilized embryo in the uterus signals to the vulva where DAF-16 is activated and induces the expression of sod-3, and potentially other unidentified targets. This leads to the production of a secondary signal that restores the HSR and stress resilience, and limits protein aggregation in somatic tissues, in an HSF-1-dependent manner. Our data suggest that a DAF-16-independent signaling route also plays a role in the restoration of the HSR (gray arrow). Error bars represent SEM. Statistical significance based on multiple $t$-tests with Holm-Sidak correction $(A, B, E)$, or two-way ANOVA followed by Sidak correction and pairwise comparisons $(C, D) .\left(^{*}\right) P<0.05 ;\left(^{* *}\right) P<0.01 ;\left(^{* *}\right) P<0.001$. $n$ denotes biological replicates of population analysis.

commitment to reproduction is detrimental to somatic health. Here, we show that progeny quality also regulates proteostasis and stress resilience in reproductive mothers. This represents a previously undescribed mode of transgenerational regulation in which compromised embryo integrity triggers a protective response in the parent that prevents the decline of the HSR and thereby improves somatic proteostasis.

Transcellular signaling pathways coordinate cell stress responses and organismal proteostasis regulation in response to a variety of physiological and environmental cues (Sala et al. 2017; Morimoto 2019). The cell-nonautonomous regulation of the HSR and unfolded protein responses can occur through neuronal control (Prahlad et al. 2008; Taylor and Dillin 2013; Williams et al. 2014; Tatum et al. 2015), between somatic tissues (van Oosten-Hawle et al. 2013; O'Brien et al. 2018), and from the germline to the soma (Shemesh et al. 2013; Labbadia and Morimoto 2015). While our previous studies indicated that repression of the HSR occurs at reproductive maturity in response to signals from the GSC (Labbadia and Morimoto 2015), we now demonstrate that the HSR and proteostasis are restored upon damage to the vitelline layer of the developing embryo in the uterus of reproductive animals. Previous studies in C. elegans have shown that DNA damage in germ cells also triggers a systemic response that relies on innate immunity and elevated proteasome activity in somatic tissues to promote organismal stress resistance (Ermolaeva et al. 2013). While DNA damage likely affects every cell type of the germline, our study specifically links the quality of the fertilized embryo to parental stress resilience. By coordinating the status of the developing proge- ny with somatic maintenance, the embryo-to-mother pathway described here is consistent with the disposable soma theory of aging, which states that allocation of organismal resources is prioritized to maximize fitness by offspring production, and that maintenance of the soma is only ensured until successful passage of the genetic material to the next generation (Kirkwood 1977). Increased somatic stress resilience in response to compromised embryos could therefore represent a strategy to reassess commitment to reproduction, perhaps to promote survival of the animal until conditions for successful reproduction are met.

We show that inhibiting the expression of either $c b d-1$, perm-2, or perm-4-the three genes known to encode proteins that reside within the vitelline layer of the eggshell -improves stress resilience and enhances proteostasis in the somatic tissues of the parent. In contrast, knockdown of genes involved in the formation of the chitin layer of the eggshell did not improve maternal stress resilience, suggesting that the embryo-to-mother pathway is specific to the vitelline layer. In all metazoans, the fertilized egg is coated with an extracellular matrix that provides physical protection to the developing embryo, known as the eggshell in C. elegans (Stein and Golden 2018). The eggshell is composed of six different layers, of which the vitelline layer is established first and is required for the proper assembly of the subsequent layers (Johnston et al. 2010). The observation that inhibiting the formation of the vitelline layer but not the chitin layer improves stress resilience is in agreement with the fact that sterility per se does not prevent the decline of the HSR, as mutants devoid of eggs or sperm still undergo repression of the 
HSR (Shemesh et al. 2013; Labbadia and Morimoto 2015). Indeed, while knockdown of either vitelline or chitin layer components compromises reproduction, the beneficial effects on somatic proteostasis are specific to disruption of the vitelline layer. This suggests that a signal released upon damage to the vitelline layer is responsible for the organismal response. CBD-1, PERM-2, and PERM4 form a multimeric protein complex in the vitelline layer of the eggshell (González et al. 2018), which may directly signal to adjacent maternal tissues to initiate the transcellular pathway upon perturbation. Alternatively, since disruption of the vitelline layer compromises the eggshell as a whole, we can speculate that exposure of the embryo plasma membrane to the uterine environment allows the release of a signal that is normally blocked by the eggshell (e.g., hormone, extracellular vesicles, or a stress signal such as reactive oxygen species). Finally, because disruption of the vitelline layer has the most deleterious consequence on eggshell structure (Johnston et al. 2010), loss of integrity of the embryo surface could be sensed mechanically by surrounding tissues.

The embryo-to-mother pathway described here involves two conserved cell stress response transcription factors, HSF-1 and DAF-16/FOXO, that are central to organismal stress resilience and longevity. Our data suggest that damage to the vitelline layer of the eggshell acts via HSF-1 in the somatic tissues of the parent to increase stress resilience. Whether vitelline damage reverses the repression that occurs at reproductive maturity via the accumulation of repressive chromatin marks at heat-shock gene loci (Labbadia and Morimoto 2015) or acts via a distinct mechanism to improve the HSR remains to be determined. We report a vulva-specific role for DAF-16 upon damage to the vitelline layer of the embryo, which is distinct from the systemic activation of DAF-16 during reduced IIS (Hsu et al. 2003), and likely to be an IIS-independent process. The localized DAF-16 response suggests that vulva tissues could monitor embryo integrity to detect and relay damage. How DAF-16 initiates the transcellular response that leads to improved stress resistance, and to what extent DAF-16 in the vulva acts upstream of HSF-1 in somatic tissues, remain to be elucidated. We propose that impairment of the vitelline layer signals to the vulva where it activates a DAF-16-dependent stress response, which could subsequently release secondary signals to other somatic tissues leading to organismal stress resilience (Fig. 5F).

The identification of a transgenerational embryo-tomother pathway that communicates progeny health to the reproductive adult to prolong the period of stress resilience and proteostasis provides additional insight into the regulation of cell protective stress responses for organismal biology, to adapt not only to environmental and physiological cues, but also to the quality of the developing progeny. Our work also uncovered a new role for the extracellular vitelline layer of the fertilized embryo as an interface in the communication of its health status to influence maternal resilience, which may be shared with other metazoans in which vitelline structures are functionally conserved.

\section{Materials and methods \\ C. elegans strains and maintenance}

Standard C. elegans methods were used as described previously (Brenner 1974). Worms were maintained on solid nematode growth medium (NGM) seeded with E. coli OP50 or for RNAi experiments, HT115 transformed with appropriate plasmids. All experiments were performed at $20^{\circ} \mathrm{C}$ unless stated otherwise. The following strains were used in this study: wild-type (N2 Bristol), AM140 rmIs132 [unc-54p::Q35::YFP] I, AMJ345 jamSi2 [mex5p::rde-1(+)] II; rde-1(ne219) V, VP303 kbIs7 [nhx-2p::rde-1+ rol-6(su1006)]; rde-1(ne219) V, CB1301 unc-54(e1301) I, AM738 rmIs297[vha-6p::Q44::YFP; rol-6(su1006)], VC2258 cbd-1 (ok2913) IV, OD95 unc-119(ed3) III; ItIs37 [(pAA64) pie-1p:: mCherry::his-58 + unc-119(+)] IV; ItIs38 [pie-1p::GFP::PH (PLC1delta1) + unc-119(+)], CB4108 fog-2(q71) V, PS3551 hsf-1 (sy441) I, AM722 rmIs288[C12C8.1p::mCherry;myo-2p::CFP], CF2253 gon-2 (q388), CF1038 daf-16(mu86) I, CF1553 muIs84 [(pAD76) sod-3p::GFP + rol-6(su1006)], CF1874 daf-16(mu86) I; muIs84, CF1580 daf-2(e1370) III; muIs84, CB1370 daf-2(e1370) III, CB1309 lin-2(e1309) X, MT308 lin-7(n308) II, JU2039 mfIs70 [lin-31p::rde-1+myo2p::GFP]; rde-1(ne219) $V$, and AM1227 mfIs70 [lin-31p::rde-1 + myo2p::GFP]; rde-1(ne219) V; cbd-1(rm19) IV. Strain VC2258 was generated by the C. elegans Gene Knockout Consortium, and was backcrossed six times to the wild-type strain before use. All other strains were either generated in our laboratory or obtained from the Caenorhabditis Genetics Center (CGC). The rmIs132 [unc-54p::Q35::YFP] transgene from strain AM140 was crossed into the genetic background of tissue targeted rde-1 strains AMJ345 and VP303 to generate strains AM1223 and AM1224, respectively. The cbd-1(ok2913) allele was crossed into the sod-3p::GFP reporter strain CF1553, resulting in strain AM1225. The introduction of egfp at the $3^{\prime}$ end of $c b d-1$, and the deletion corresponding to the $c b d-1$ (ok2913) allele into the JU2039 strain, were performed using CRISPR/Cas9 genome editing and resulted in strains AM1226 cbd-1/rm18/cbd1::egfp]) IV and AM1227 mfIs70 [lin-31p::rde-1 + myo2p::GFP]; rde-1(ne219) V; cbd-1(rm19) IV, respectively (see the Supplemental Material).

\section{Genetic screen}

For the tissue targeted RNAi screen, the AMJ345 strain, which expresses the RNAi essential gene $r d e-1$ under the control of the germline promoter mex-5 in an otherwise rde-1-deficient background, was crossed into Q35::YFP(Morley et al. 2002; Marré et al. 2016), and we confirmed that the resulting strain (AM1223) was resistant to yfp RNAi (Supplemental Fig. S1A,C). Our laboratory previously identified a set of modifiers that suppress Q35:: YFP aggregation upon systemic RNAi (Silva et al. 2011). To test whether a subset of these act cell-nonautonomously from the germline, we used an RNAi library of 93 clones that correspond to the modifiers that suppressed aggregation of both Q35::YFP

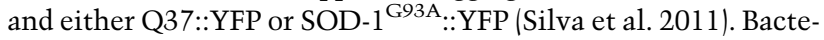
ria were inoculated in LB containing Ampicillin in a 96-well imaging plate (Falcon), grown overnight at $37^{\circ} \mathrm{C}$, and production of dsRNA was induced with $5 \mathrm{mM}$ IPTG for $3 \mathrm{~h}$. Synchronized L1 stage animals were added $(\sim 15$ per well $)$ and incubated at $20^{\circ} \mathrm{C}$ with gentle shaking. At day 3 of adulthood ( $5 \mathrm{~d}$ after L1 stage), animals were immobilized by adding $2 \mathrm{mM}$ levamisole to the wells and $\mathrm{Z}$ stacks were captured using an ImageXpress confocal microscope with a $\times 10$ objective. Q35::YFP foci per animals were visually counted from maximum intensity projections generated from the confocal images. Aggregates were defined as bright fluorescent foci clearly distinct from background YFP fluorescence. 
This analysis resulted in 35 candidates that significantly reduced the number of Q35::YFP aggregates as compared with the empty vector control ( $t$-test $P$-value $<0.01$ in two rounds) (Supplemental Fig. S1E). The AMJ345 strain was shown to have leaky RNAi activity in the intestine (Marré et al. 2016), and we thus performed a counter-screen using the strain AM1224, which results from crossing VP303, in which RNAi is restricted to the intestine (nhx-2 promoter driving $r d e-1$ expression) to Q35::YFP (validation of the strain shown in Supplemental Fig. S1B,D). Using the same procedure, we found 19 intestinal modifiers of muscle Q35::YFP aggregation (Supplemental Fig. S1E). After subtracting the intestinal modifiers from the candidates obtained in the initial screen, as well as RNAi clones that led to severe growth defects, we performed two rounds of validation on NGM plates in the AM1223 strain, which resulted in six final germline hits (Supplemental Fig. S1F). The genetic screen, counter-screen, and validation were performed in a blinded manner with respect to RNAi condition.

\section{RNAi treatments}

For RNAi-mediated knockdown of indicated genes, synchronized L1 stage animals were plated on E. coli strain HT115(DE3) containing the appropriate RNAi vectors obtained from the Ahringer RNAi library and using L4440 as the empty vector control (Kamath et al. 2003). To prepare RNAi NGM plates, overnight cultures were diluted $1 / 50$ in the morning, allowed to grow for $3 \mathrm{~h}$ at $37^{\circ} \mathrm{C}$, and induced with $5 \mathrm{mM} \mathrm{IPTG}$ for $3 \mathrm{~h}$.

\section{Quantification of polyglutamine aggregates}

Populations of at least 25 synchronized animals were grown at $20^{\circ} \mathrm{C}$ until the day of adulthood indicated, transferred to fresh plates, and the number of polyQ::YFP aggregate per animal was visually determined using a fluorescence microscope. Aggregates were defined as bright fluorescent foci clearly distinct from background YFP fluorescence. Experiments were repeated three times.

\section{Fluorescence microscopy}

For epifluorescence images, age-synchronized animals were mounted on $3 \%$ agarose pads in $2 \mathrm{mM}$ levamisole and imaged with a Zeiss Axiovert 200 microscope using either a 10x or 40x objective lens. Images were captured using Zeiss Axiovision software and processed in Image $(\mathrm{NIH})$. For confocal microscopy, age-synchronized animals were mounted on $3 \%$ agarose pads in $25 \mathrm{mM}$ sodium azide and imaged with a Zeiss LSM 800 confocal microscope using a $10 \times$ or $20 \times$ objective lens and the Zen imaging software. For the sod-3p::GFP reporter analysis, maximum intensity projection images were generated from $\mathrm{Z}$ stacks and fluorescence intensity was measured by tracing either whole animals or regions of interest using ImageJ software. The "head" region was defined from the nose to the beginning of the intestine and the "tail" as the posterior region down from the vulva (excluded).

Heat stress survival assay

Synchronized animals (50 per plate) were grown at $20^{\circ} \mathrm{C}$ until the day of adulthood indicated, transferred to new plates, sealed with parafilm and incubated for $4 \mathrm{~h}$ in a water bath equilibrated at $35^{\circ} \mathrm{C}$. Worms were allowed to recover for $48 \mathrm{~h}$ at $20^{\circ} \mathrm{C}$, or $72 \mathrm{~h}$ for gravid day 1 analysis, and scored for survival. Animals were scored as dead in the absence of pharyngeal pumping or touch re- sponse, and animals with prolapsed gonads were censored from the analysis. Experiments were repeated at least three times.

Heat shock for expression analysis

Plates containing $\sim 100$ age-synchronized animals were wrapped with parafilm and heat shocked in a water bath for $30 \mathrm{~min}$ at $33^{\circ} \mathrm{C}$. Animals were collected immediately following heat shock and snap-frozen in liquid nitrogen. Heat-shock experiments and real-time qPCR were performed at least in triplicate.

\section{$R N A$ extraction and real-time $q P C R$}

RNA extraction was performed on populations of at least 100 animals per replicate using TRIzol (Invitrogen) and RNA purified with QIAGEN RNeasy MinElute columns as described previously (Labbadia and Morimoto 2015). mRNA was reverse transcribed using the iScript cDNA synthesis kit (Bio-Rad) and real-time quantitative PCR was performed using iQ SYBR Green Supermix (Bio-Rad) in a Bio-Rad CFX384 real-time PCR system. Relative expression was determined from cycle threshold values using the standard curve method and the expression of genes of interest was normalized to $c d c-42$. The primers used are listed in Supplemental Table S1.

\section{Statistical analyses}

For all experiments, at least three independent biological replicates were performed unless stated otherwise. Data are presented as mean values \pm SEM. $P$-values were calculated using GraphPad Prism with either one-way ANOVA with Dunnett correction and pairwise comparison of groups, two-way ANOVA followed by Sidak correction for pairwise comparisons, multiple $t$-tests with Holm-Sidak correction, or two-tailed Student's $t$-test as stated in the figure legends. Single asterisks denote $P<0.05$, double asterisks denote $P<0.01$, triple asterisks denote $P<0.001$, and "ns" stands for nonsignificant and denotes $P>0.05$.

\section{Acknowledgments}

We thank John Labbadia for discussions on the RNAi screening strategy and heat stress assays, Erin Aprison for assistance with germline staining experiments, and Ilya Ruvinsky, Anan Yu, and Thomas Stoeger for critical reading of the manuscript. We also thank the Keck Biophysics and High-Throughput Analysis facilities as well as the laboratory of Robert Lamb at Northwestern University for access to equipment. This work was supported by grants from the National Institutes of Health (National Institute on Aging R56AG059579, R37AG026647, RF1AG057296, and P01AG054407) and the Daniel F. and Ada L. Rice Foundation to R.I.M., and by postdoctoral fellowships from the National Ataxia Foundation and the American Federation for Aging Research as well as a research grant from the Kennedy's Disease Association to L.C.B.

Author contributions: A.J.S. and R.I.M. conceived the study. A.J.S., L.C.B., and R.I.M. designed the experiments. A.J.S. and R.M.B. performed the experiments. A.J.S. and R.I.M. analyzed the data. A.J.S, L.C.B., and R.I.M. wrote the manuscript.

\section{References}

Antebi A. 2013. Regulation of longevity by the reproductive system. Exp Gerontol 48: 596-602. doi:10.1016/j.exger.2012.09 .009 
Barkoulas M, van Zon JS, Milloz J, van Oudenaarden A, Félix MA. 2013. Robustness and epistasis in the C. elegans vulval signaling network revealed by pathway dosage modulation. Dev Cell 24: 64-75. doi:10.1016/j.devcel.2012.12.001

Ben-Zvi A, Miller EA, Morimoto RI. 2009. Collapse of proteostasis represents an early molecular event in Caenorhabditis elegans aging. Proc Natl Acad Sci 106: 14914-14919. doi:10 $.1073 /$ pnas.0902882106

Brenner S. 1974. The genetics of Caenorhabditis elegans. Genetics 77: 71-94.

Ermolaeva MA, Segref A, Dakhovnik A, Ou HL, Schneider JI, Utermöhlen O, Hoppe T, Schumacher B. 2013. DNA damage in germ cells induces an innate immune response that triggers systemic stress resistance. Nature 501: 416-420. doi:10 $.1038 /$ nature 12452

Espelt MV, Estevez AY, Yin X, Strange K. 2005. Oscillatory Ca ${ }^{2+}$ signaling in the isolated Caenorhabditis elegans intestine: role of the inositol-1,4,5-trisphosphate receptor and phospholipases C $\beta$ and $\gamma$. J Gen Physiol 126: 379-392. doi:10.1085/igp .200509355

Ferguson EL, Horvitz HR. 1985. Identification and characterization of 22 genes that affect the vulval cell lineages of the nematode Caenorhabditis elegans. Genetics 110: 17-72.

Flatt T, Min KJ, D'Alterio C, Villa-Cuesta E, Cumbers J, Lehmann R, Jones DL, Tatar M. 2008. Drosophila germ-line modulation of insulin signaling and lifespan. Proc Natl Acad Sci 105: 6368-6373. doi:10.1073/pnas.0709128105

Gidalevitz T, Ben-Zvi A, Ho KH, Brignull HR, Morimoto RI. 2006. Progressive disruption of cellular protein folding in models of polyglutamine diseases. Science 311: 1471-1474. doi:10.1126/science.1124514

González DP, Lamb HV, Partida D, Wilson ZT, Harrison MC, Prieto JA, Moresco JJ, Diedrich JK, Yates JR III, Olson SK. 2018. CBD-1 organizes two independent complexes required for eggshell vitelline layer formation and egg activation in $C$. elegans. Dev Biol 442: 288-300. doi:10.1016/j.ydbio.2018.08 .005

Gupta BP, Hanna-Rose W, Sternberg PW. 2012. Morphogenesis of the vulva and the vulval-uterine connection. In WormBook (ed. The C. elegans Research Community), WormBook. doi:10 .1895/wormbook.1.152.1, http://www.wormbook.org

Hipp MS, Kasturi P, Hartl FU. 2019. The proteostasis network and its decline in ageing. Nat Rev Mol Cell Biol 20: 421435. doi:10.1038/s41580-019-0101-y

Hsin H, Kenyon C. 1999. Signals from the reproductive system regulate the lifespan of C. elegans. Nature 399: 362-366. doi: $10.1038 / 20694$

Hsu AL, Murphy CT, Kenyon C. 2003. Regulation of aging and age-related disease by DAF-16 and heat-shock factor. Science 300: 1142-1145. doi:10.1126/science.1083701

Inoue T, Sherwood DR, Aspöck G, Butler JA, Gupta BP, Kirouac M, Wang M, Lee PY, Kramer JM, Hope I, et al. 2002. Gene expression markers for Caenorhabditis elegans vulval cells. Mech Dev 119: S203-S209. doi:10.1016/S0925-4773(03) 00117-5

Johnston WL, Krizus A, Dennis JW. 2006. The eggshell is required for meiotic fidelity, polar-body extrusion and polarization of the C. elegans embryo. BMC Biol 4: 35. doi:10.1186/17417007-4-35

Johnston WL, Krizus A, Dennis JW. 2010. Eggshell chitin and chitin-interacting proteins prevent polyspermy in C. elegans. Curr Biol 20: 1932-1937. doi:10.1016/j.cub.2010.09.059

Kamath RS, Fraser AG, Dong Y, Poulin G, Durbin R, Gotta M, Kanapin A, Le Bot N, Moreno S, Sohrmann M, et al. 2003. Systematic functional analysis of the Caenorhabditis elegans ge- nome using RNAi. Nature 421: 231-237. doi:10.1038/ nature 01278

Kirkwood TB. 1977. Evolution of ageing. Nature 270: 301-304. doi:10.1038/270301a0

Korennykh A, Walter P. 2012. Structural basis of the unfolded protein response. Annu Rev Cell Dev Biol 28: 251-277. doi:10.1146/annurev-cellbio-101011-155826

Labbadia J, Morimoto RI. 2015. Repression of the heat shock response is a programmed event at the onset of reproduction. Mol Cell 59: 639-650. doi:10.1016/j.molcel.2015.06.027

Li J, Labbadia J, Morimoto RI. 2017. Rethinking HSF1 in stress, development, and organismal health. Trends Cell Biol 27: 895-905. doi:10.1016/j.tcb.2017.08.002

Libina N, Berman JR, Kenyon C. 2003. Tissue-specific activities of C. elegans DAF-16 in the regulation of lifespan. Cell 115: 489-502. doi:10.1016/S0092-8674(03)00889-4

Marré J, Traver EC, Jose AM. 2016. Extracellular RNA is transported from one generation to the next in Caenorhabditis elegans. Proc Natl Acad Sci 113: 12496-12501. doi:10.1073/pnas .1608959113

Maruyama R, Velarde NV, Klancer R, Gordon S, Kadandale P, Parry JM, Hang JS, Rubin J, Stewart-Michaelis A, Schweinsberg P, et al. 2007. EGG-3 regulates cell-surface and cortex rearrangements during egg activation in Caenorhabditis elegans. Curr Biol 17: 1555-1560. doi:10.1016/j.cub.2007.08.011

Morimoto RI. 2019. Cell-nonautonomous regulation of proteostasis in aging and disease. Cold Spring Harb Perspect Biol doi:10.1101/cshperspect.a034074

Morley JF, Morimoto RI. 2004. Regulation of longevity in Caenorhabditis elegans by heat shock factor and molecular chaperones. Mol Biol Cell 15: 657-664. doi:10.1091/mbc.e03-070532

Morley JF, Brignull HR, Weyers JJ, Morimoto RI. 2002. The threshold for polyglutamine-expansion protein aggregation and cellular toxicity is dynamic and influenced by aging in Caenorhabditis elegans. Proc Natl Acad Sci 99: 1041710422. doi:10.1073/pnas.152161099

O'Brien D, Jones LM, Good S, Miles J, Vijayabaskar MS, Aston R, Smith CE, Westhead DR, van Oosten-Hawle P. 2018. A PQM1-mediated response triggers transcellular chaperone signaling and regulates organismal proteostasis. Cell Rep 23: 3905-3919. doi:10.1016/j.celrep.2018.05.093

Oh SW, Mukhopadhyay A, Dixit BL, Raha T, Green MR, Tissenbaum HA. 2006. Identification of direct DAF-16 targets controlling longevity, metabolism and diapause by chromatin immunoprecipitation. Nat Genet 38: 251-257. doi:10.1038/ ng1723

Olson SK, Greenan G, Desai A, Müller-Reichert T, Oegema K. 2012. Hierarchical assembly of the eggshell and permeability barrier in C. elegans. J Cell Biol 198: 731-748. doi:10.1083/jcb .201206008

Owusu-Ansah E, Song W, Perrimon N. 2013. Muscle mitohormesis promotes longevity via systemic repression of insulin signaling. Cell 155: 699-712. doi:10.1016/j.cell.2013.09.021

Prahlad V, Cornelius T, Morimoto RI. 2008. Regulation of the cellular heat shock response in Caenorhabditis elegans by thermosensory neurons. Science 320: 811-814. doi:10.1126/ science. 1156093

Sala AJ, Bott LC, Morimoto RI. 2017. Shaping proteostasis at the cellular, tissue, and organismal level. I Cell Biol 216: 12311241. doi:10.1083/jcb. 201612111

Schedl T, Kimble J. 1988. fog-2, a germ-line-specific sex determination gene required for hermaphrodite spermatogenesis in Caenorhabditis elegans. Genetics 119: 43-61. 
Sharma-Kishore R, White JG, Southgate E, Podbilewicz B. 1999. Formation of the vulva in Caenorhabditis elegans: a paradigm for organogenesis. Development 126: 691-699.

Shemesh N, Shai N, Ben-Zvi A. 2013. Germline stem cell arrest inhibits the collapse of somatic proteostasis early in Caenorhabditis elegans adulthood. Aging Cell 12: 814-822. doi:10 .1111 /acel.12110

Sherwood DR, Sternberg PW. 2003. Anchor cell invasion into the vulval epithelium in C. elegans. Dev Cell 5: 21-31. doi:10 $.1016 /$ S1534-5807/03/00168-0

Shpilka T, Haynes CM. 2018. The mitochondrial UPR: mechanisms, physiological functions and implications in ageing. Nat Rev Mol Cell Biol 19: 109-120. doi:10.1038/nrm.2017.110

Silva MC, Fox S, Beam M, Thakkar H, Amaral MD, Morimoto RI. 2011. A genetic screening strategy identifies novel regulators of the proteostasis network. PLoS Genet 7: e1002438. doi:10 .1371/journal.pgen.1002438

Simske JS, Kaech SM, Harp SA, Kim SK. 1996. LET-23 receptor localization by the cell junction protein LIN-7 during C. elegans vulval induction. Cell 85: 195-204. doi:10.1016/S00928674(00)81096-X

Stein KK, Golden A. 2018. The C. elegans eggshell. WormBook 2018: 1-36. doi:10.1895/wormbook.1.179.1

Sternberg PW, Horvitz HR. 1986. Pattern formation during vulval development in C. elegans. Cell 44: 761-772. doi:10.1016/ 0092-8674(86)90842-1

Sternberg PW, Horvitz HR. 1989. The combined action of two intercellular signaling pathways specifies three cell fates during vulval induction in C. elegans. Cell 58: 679-693. doi:10.1016/ 0092-8674/89) $90103-7$

Tatum MC, Ooi FK, Chikka MR, Chauve L, Martinez-Velazquez LA, Steinbusch HWM, Morimoto RI, Prahlad V. 2015. Neuronal serotonin release triggers the heat shock response in $C$. elegans in the absence of temperature increase. Curr Biol 25: 163-174. doi:10.1016/j.cub.2014.11.040

Taylor RC, Dillin A. 2013. XBP-1 is a cell-nonautonomous regulator of stress resistance and longevity. Cell 153: 1435-1447. doi:10.1016/j.cell.2013.05.042

van Oosten-Hawle P, Porter RS, Morimoto RI. 2013. Regulation of organismal proteostasis by transcellular chaperone signaling. Cell 153: 1366-1378. doi:10.1016/j.cell.2013.05.015

Walther DM, Kasturi P, Zheng M, Pinkert S, Vecchi G, Ciryam P, Morimoto RI, Dobson CM, Vendruscolo M, Mann M, et al. 2015. Widespread proteome remodeling and aggregation in aging C. elegans. Cell 161: 919-932. doi:10.1016/j.cell.2015.03 .032

Williams KW, Liu T, Kong X, Fukuda M, Deng Y, Berglund ED, Deng Z, Gao Y, Liu T, Sohn JW, et al. 2014. Xbp1s in Pomc neurons connects ER stress with energy balance and glucose homeostasis. Cell Metab 20: 471-482. doi:10.1016/j.cmet .2014 .06 .002

Zhang P, Judy M, Lee SJ, Kenyon C. 2013. Direct and indirect gene regulation by a life-extending FOXO protein in C. elegans: roles for GATA factors and lipid gene regulators. Cell Metab 17: 85-100. doi:10.1016/j.cmet.2012.12.013 


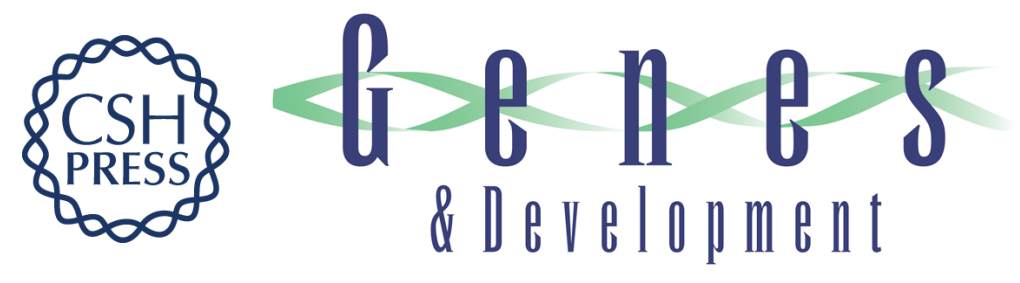

\title{
Embryo integrity regulates maternal proteostasis and stress resilience
}

\author{
Ambre J. Sala, Laura C. Bott, Renee M. Brielmann, et al.
}

Genes Dev. 2020, 34: originally published online March 26, 2020

Access the most recent version at doi:10.1101/gad.335422.119

\section{Supplemental Material \\ http://genesdev.cshlp.org/content/suppl/2020/03/25/gad.335422.119.DC1 \\ References This article cites 50 articles, 16 of which can be accessed free at: http://genesdev.cshlp.org/content/34/9-10/678.full.html\#ref-list-1 \\ Creative This article, published in Genes \& Development, is available under a Creative Commons Commons License (Attribution-NonCommercial 4.0 International), as described at License http://creativecommons.org/licenses/by-nc/4.0/. \\ Email Alerting Receive free email alerts when new articles cite this article - sign up in the box at the top Service right corner of the article or click here.}

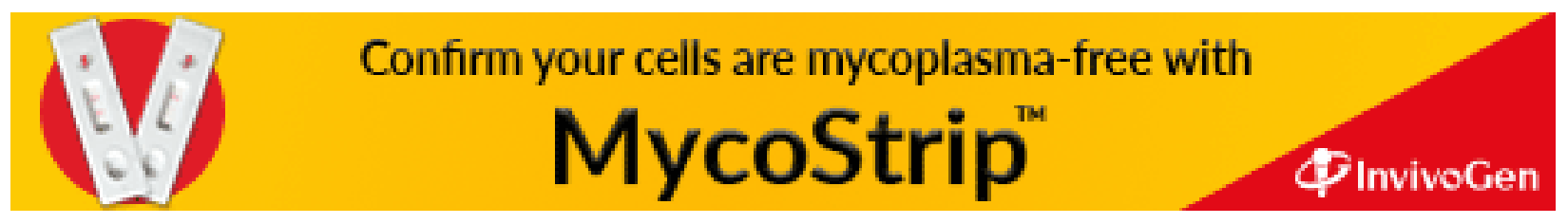

